\title{
THE CORRESPONDING POINTS SCREENING ALGORITHM BASED ON GAUSSIAN KERNEL FUZZY CLUSTERING
}

\author{
Q. Tan ${ }^{1,2}$, H. Zhao ${ }^{1,2 *}$, W. Wu ${ }^{1,2}$ \\ ${ }^{1}$ Department of Civil Engineering, Tsinghua University, Beijing 10084, China \\ - (tqf17, zhr,wu-wj14)@mails.tsinghua.edu.cn \\ ${ }^{2} 3 \mathrm{~S}$ Center, Tsinghua University, Beijing 10084, China
}

ICWG II/III: Pattern Analysis in Remote Sensing

\begin{abstract}
KEY WORDS: Corresponding Points Matching, Gaussian Kernel, Fuzzy Clustering, High-dimensional Feature Space ABSTRACT:

Corresponding points matching is the basis of three-dimensional reconstruction, but mismatching often occurs in feature matching. Existing algorithms for handling mismatches, such as RANSAC, mostly use the distance from the point to the polar line (i.e., the residual) to determine whether the matching relationship is correct. However, the residual cannot effectively ensure the correctness of the match. In this paper, the Gaussian kernel method is introduced to map the one-dimensional indivisible residual to the highdimensional feature space, and the inliers and the outliers are distinguished by fuzzy clustering. After simulation data and actual image data verification, the proposed algorithm has significant improvement in accuracy and efficiency compared with the traditional RANSAC algorithm.
\end{abstract}

\section{INTRODUCTION}

Feature matching for correspondence is posed as the following problem - it extracts pairs of feature points describing same scenes, which are also called corresponding points, in two images The method is based on the following hypothesis: the corresponding points have approximate image features. Therefore, by comparing the similarity of feature descriptors, the corresponding points can be preliminarily screened, in which the Scale Invariant Feature Transform (SIFT) descriptor is most used Compared with grey matching and transform domain-based matching, feature matching method is more robust to noise, occlusion, illumination and image distortion, so it has been widely applied in three-dimensional reconstruction, image registration, image mosaic and so on. However, due to the small amount of information used in feature matching, errors are inevitable in matching results.

In order to solve the problem of matching errors, many solutions have been proposed, which can be divided into three categories. The first type of methods reduces errors by improving feature descriptors. For example, in 2014, Ye et al. used the improved SR-SIFT descriptor based on SIFT to characterize the feature, which can eliminate the difference of translation, rotation and scale between images. Then they added the information of Harris operator and the local self-similarity descriptor to promote the precision (Ye et al., 2014). Generally, this type of methods significantly increases the amount of calculation, while the result is not significantly improved.

The second type introduces new information to the match process. In 2016, Li used image segmentation to aid SIFT feature matching. The images were segmented into many blocks, and the similar blocks were matched by comparing the image energy. That means corresponding points should be in similar blocks, effectively reducing the probability of errors $(\mathrm{Li}, 2016)$. These methods usually improve the computational efficiency and matching accuracy, but relies on the prior knowledge. The degree of automation and the robustness of the algorithm need to be improved.

The third type improves the feature matching algorithm. Currently the most commonly used algorithm is the random sample consensus algorithm (RANSAC), which is proposed by Fischer and Bolles in 1981. The preliminary matching results are divided into inliers and outliers, representing correct and wrong corresponding points. The random sample is continuously executed until the probability that samples are all inliers reaches the confidence level. RANSAC can effectively process data containing a large number of outliers, but the time consumption increases dramatically as the outliers increase. Besides, due to the randomness of sampling, the results of a single experiment cannot be reproduced. In recent decades, many scholars have proposed improvements to the RANSAC, such as GOODSAC and SURESAC, but the improvement results are limited.

Referring to validation, traditional methods mainly use residual, namely the distance from the feature point to the polar line, to judge whether the matching relationship is correct. If the residual is smaller than our experimental threshold, the corresponding points are considered to be inliers, otherwise to be outliers. However, the criterion is not rigorous, since outliers may also have small residuals. In fact, the distributions of the inliers' and outliers' residuals are not independent, but have overlapping parts. But as far as I know, currently few studies take this issue into consideration. Therefore, new methods and new criteria are expected to accurately identify corresponding points.

The kernel function increases the probability of data separability by mapping the data to a higher-dimensional space. It has been used in common algorithms, such as principal component analysis (PCA) and support vector machines (SVM), to play its classification role. In this paper, the idea of kernel function is introduced to map the one-dimensional residual feature to the high-dimensional feature space, and then the linear division of

\footnotetext{
* Corresponding author, Email: zhr@tsinghua.edu.cn.
} 
the inliers and outliers is carried out so that correct corresponding points can be screened.

\section{THEORIES}

\subsection{The Gaussian Kernel}

The kernel function is capable of mapping low dimensional spatial data to high dimensional space, so that the linear classification mode can be obtained by methods such as support vector machine. The advantage of kernel function is that the inner product can be used as the input function rather than explicitly calculating the mapping function, reducing the amount of computation. Therefore, the kernel function can effectively assist to distinguish many data that cannot be linearly classified in lowdimensional space.

The Gaussian kernel is one of the most widely used kernel functions, which can be regarded as a kernel of infinite power polynomial. Thus, it can be considered that the feature space is infinitely dimensioned after Gaussian kernel mapping, while the number of matching points we have calculated is limited. Dividing finite-dimensional data in an infinite dimensional space, can always obtain linear separable results theoretically. In this paper, Gaussian kernel function and SVM are utilized to screen correct corresponding points.

The Gaussian kernel expression is:

$$
\mathrm{K}(\mathrm{x}, \mathrm{y})=\exp \left(-\frac{\|x-y\|^{2}}{2 \sigma^{2}}\right) \text {. }
$$

Where $\sigma$ controls the flexibility of the kernel. If the value of $\sigma$ is too small, there is a risk of overfitting. On the contrary, if the value is too big, the kernel will gradually be reduced to a constant function and lose the classification ability.

After the Gaussian space mapping is performed on the residuals of the matching points, the distance from the residual to the cluster center is:

$$
d_{j i}=\sqrt{2-2 K\left(x_{i}, \mu_{j}\right)} .
$$

Where $d_{j i}$ is the distance between the $\mathrm{i}$-th residual and the $\mathrm{j}$-th cluster center in the feature space. In this paper, the matching points are clustered into inliers and outliers, so $\mathrm{j}$ is 1 or 2 .

\subsection{Fuzzy Clustering}

The fuzzy clustering method clusters the samples by comparing the distance between samples and cluster centers, and the cluster centers are randomly determined under a specific objective function. Then, the centers are updated according to the clustering result. The algorithm is repeated until the iteration termination threshold is reached.

In the residual fuzzy clustering experiment, samples are the residuals of the matching points. Suppose $X=\left\{x_{i}, i=1,2, \cdots, n\right\}$ is a sample set, and $\mu_{\mathrm{j}}(\mathrm{j}=1,2)$ are 2 cluster centers, the objective function of fuzzy clustering is:

$$
\mathrm{F}=\sum_{j=1}^{2} \sum_{i=1}^{n} P\left(\mu_{j} \mid x_{i}\right)^{2} d_{j i} .
$$

The purpose of clustering is to minimize the objective function $\mathrm{F}$. As mentioned in 2.1, $\mathrm{d}_{\mathrm{ji}}$ is the distance from the residual to the cluster center, which can be calculated by the equation (1). $\mathrm{P}\left(\mu_{\mathrm{j}} \mid \mathrm{x}_{\mathrm{i}}\right)$ is the membership function of the $\mathrm{i}$-th residual to the $\mathrm{j}$-th class, which satisfies $0 \leq \mathrm{P}\left(\mu_{\mathrm{j}} \mid \mathrm{x}_{\mathrm{i}}\right) \leq 1$ and $0<\sum_{\mathrm{i}=1}^{\mathrm{n}} \mathrm{P}\left(\mu_{\mathrm{j}} \mid \mathrm{x}_{\mathrm{i}}\right)<\mathrm{n}$.

Minimize $F$ by adjusting the cluster center $\mu_{\mathrm{j}}$ and the membership function $\mathrm{P}\left(\mu_{\mathrm{j}} \mid \mathrm{x}_{\mathrm{i}}\right)$. The equations are:

$$
\begin{aligned}
& \frac{\partial \mathrm{F}}{\partial \mu_{\mathrm{j}}}=0 \\
& \frac{\partial \mathrm{F}}{\partial \mathrm{P}_{\mathrm{j}}}=0 .
\end{aligned}
$$

The solutions are:

$$
\begin{gathered}
\mu_{j}=\frac{\sum_{i=1}^{n} P\left(\mu_{j} \mid x_{i}\right)^{2} x_{i}}{\sum_{i=1}^{n} P\left(\mu_{j} \mid x_{i}\right)^{2}} \\
P\left(\mu_{j} \mid x_{i}\right)=\frac{d_{j i}^{-1}}{\sum_{j=1}^{2} d_{j i}^{-1}} .
\end{gathered}
$$

All membership functions $\mathrm{P}\left(\mu_{\mathrm{j}} \mid \mathrm{x}_{\mathrm{i}}\right)$ can form a membership matrix $\mathrm{P}$ of $\mathrm{i}$ rows and $\mathrm{j}$ columns.

By repeating the process from Equation (2) to Equation (5), the cluster center and membership function in the high-dimensional feature space can be iteratively obtained, further the classification results can be calculated.

However, in the low dimension space, although fuzzy clustering can calculate the classification results, the samples are not linearly separable. Therefore, it is also necessary to analyze the linear separability in the feature space.

\subsection{Separability analysis}

For the classified inliers and outliers, there are usually two kinds of errors: the matching points belonging to the inliers fall into the outliers set area, or the matching points belonging to the outliers fall into the inliers set. The one-dimensional case of this result is shown as below.

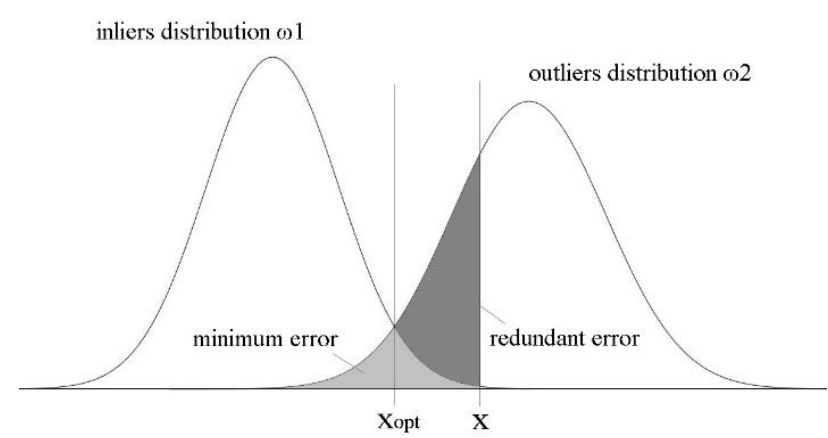

Figure 1. One-dimensional classification error case

$\omega_{1}$ and $\omega_{2}$ represent inliers and outliers distribution. $x$ represents the decision boundary for judging the inliers and outliers at this time. The shaded portion is the error rate. When the decision boundary moves to $\mathrm{x}_{\mathrm{opt}}$, the total error rate is the minimum, which is called the Bayesian error rate caused by the Bayesian 
decision. The Bayesian decision rule ensures the lowest probability of error, but it cannot quantify this minimum. The calculation of the error rate is also complicated. Fortunately, when there are only two types of classification results, the upper limit of probability can be approximated by the error integration formula. The Chernoff bound of probability $\mathrm{P}$ is chosen as the criterion for judging the clustering results.

$$
\mathrm{P} \leq \int P^{\beta}\left(x \mid \omega_{1}\right) P^{1-\beta}\left(x \mid \omega_{2}\right) d x=e^{-k(\beta)} .
$$

The Chernoff bound of $\mathrm{P}$ can be found by calculating the beta value to minimize $\mathrm{e}^{-\mathrm{k}(\beta)}$. According to the research by Duda et al. in $2003, \beta=0.66$ is selected.

After mathematical reasoning, the separability judgment function of constructing the inliers and the outliers set can be indicated as follows:

$$
\mathrm{J}=\frac{\beta(1-\beta)\left(\mu_{2}-\mu_{1}\right)^{2}}{2\left[\beta \sigma_{1}+(1-\beta) \sigma_{2}\right]}+\frac{1}{2} \ln \frac{\left|\beta \sigma_{1}+(1-\beta) \sigma_{2}\right|}{\sigma_{1}^{\beta}+\sigma_{2}^{1-\beta}}
$$

Brandt (2002) found that the residuals of the inliers and outliers satisfied different Gaussian distributions. Therefore, $\sigma_{1}$ and $\sigma_{2}$ in Equation (7) represent the standard deviation of the two distributions.

The stable value of the judgment function in the highdimensional space can be obtained by iteration, which represents the optimal classification result of the residuals.

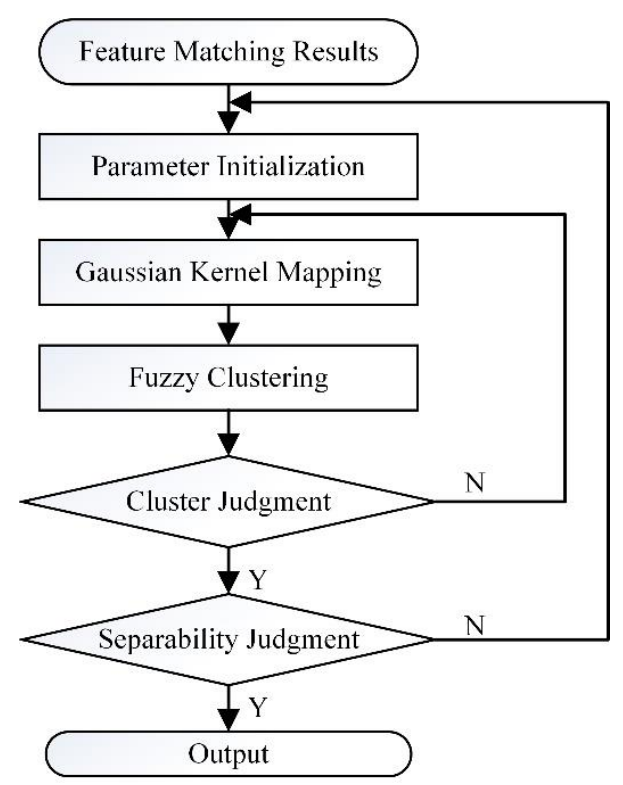

Figure 2. The Gaussian Kernel Fuzzy Clustering algorithm flowchart

In total, the steps of the Gaussian kernel fuzzy clustering algorithm can be summarized as follows:

(1) Initialize the clustering centers, and assign a random number to the membership function in the interval $[0,1]$ to obtain the fuzzy membership matrix $P$;

(2) Map the matching point residuals by the Gaussian kernel to obtain the distance of the residual in the feature space;

(3) Calculate new cluster centers and the fuzzy membership matrix according to equation (5);

(4) Calculate the amount of change in the cluster centers and the fuzzy membership matrix. If the amount is less than a certain threshold, the algorithm terminates. Otherwise repeat steps (2) and (3);

(5) Calculate the clustering result to obtain the inliers and the outliers. Determine whether the function in Equation (7) tend to be stable. If it changes little with iteration, output the result. Otherwise, return to step (1).

\section{EXPERIMENTS}

Three sets of experiments were designed to verify the efficiency, accuracy and robustness of the proposed algorithm using simulated and actual image data. At the same time, the RANSAC algorithm were selected for comparative analysis. Since the results of the RANSAC algorithm are non-repeatable, the data results used for comparison are the average results of multiple experiments.

\subsection{Efficiency verification}

200 pairs of corresponding points are simulated, and then some random mismatching points are added, so that the initial error rate is $5 \%, 10 \%, \ldots, 50 \%$. The proposed algorithm and RANSAC algorithm are respectively applied to find inliers. And the confidence level of RANSAC is set to $99.9 \%$. The time consumption of the two methods are compared at different outlier rates.

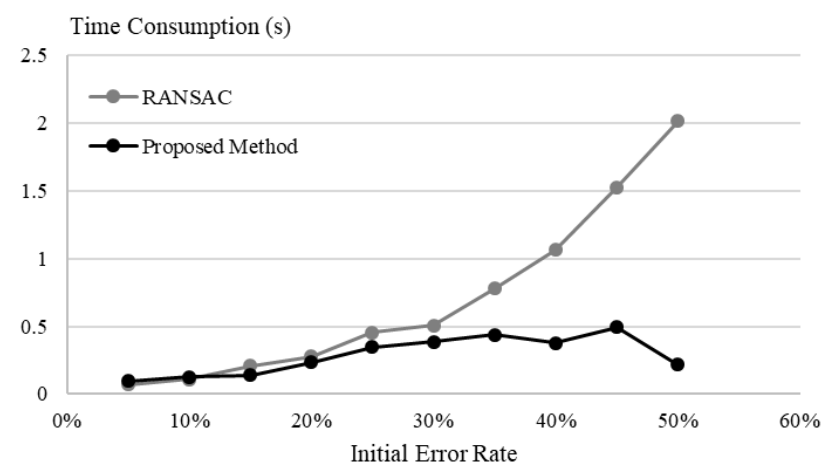

Figure 3. Time consumption of two algorithms

The results show that when the outlier rate is less than $10 \%$, the time consumption of the proposed algorithm is slightly higher than that of the RANSAC algorithm. As the rate increases, the time of the RANSAC algorithm increases rapidly, while that of the proposed algorithm changes little. When the error rate exceeds $30 \%$, the proposed algorithm shows a significant efficiency advantage. In addition, the calculation of the RANSAC requires initial error rate, which is unknown in actual experiments.

\subsection{Accuracy verification}

Considering the actual image, the experimental accuracy is affected not only by the initial matching error, but also the noise. Therefore, based on the previous experiment, the initial error rate is set to $10 \%$ (average error level), and the Gaussian noise with a standard deviation of $0.5,1, \ldots, 5$ pixels was added. The accuracy of the proposed method and the RANSAC method for different variances are compared.

The results show that the accuracy of both algorithms will decrease as the standard deviation of noise increases. As the impact of noise increases, there is no guarantee that the outliers will be completely eliminated in each calculation, so the accuracy is continuously declining. Comparing the two algorithms, the 
proposed algorithm has higher precision and robustness. When the noise is less than 3 pixels, the classification accuracy exceeds $90 \%$.

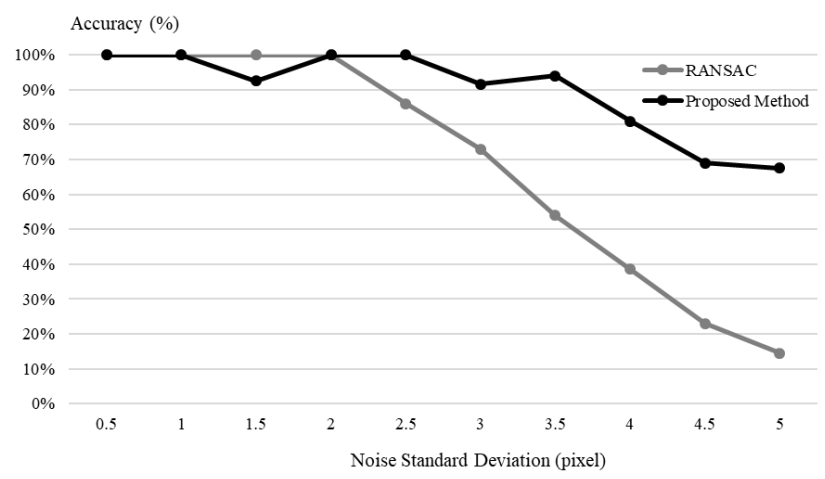

Figure 4. Accuracy comparison of two algorithms for processing noisy data

\subsection{Actual image experiments}

Besides simulation experiments, actual image experiments are also performed for verification. Taking two images of a rabbit doll, the preliminary feature matching obtained 372 pairs of points. Wire the matching points of the two images, a small number of mismatched points can be find. The inliers and outliers are separated by the proposed algorithm and the RANSAC algorithm respectively. Assuming that the external point ratio of the RANSAC algorithm is $20 \%$, the confidence is set to $99.9 \%$.

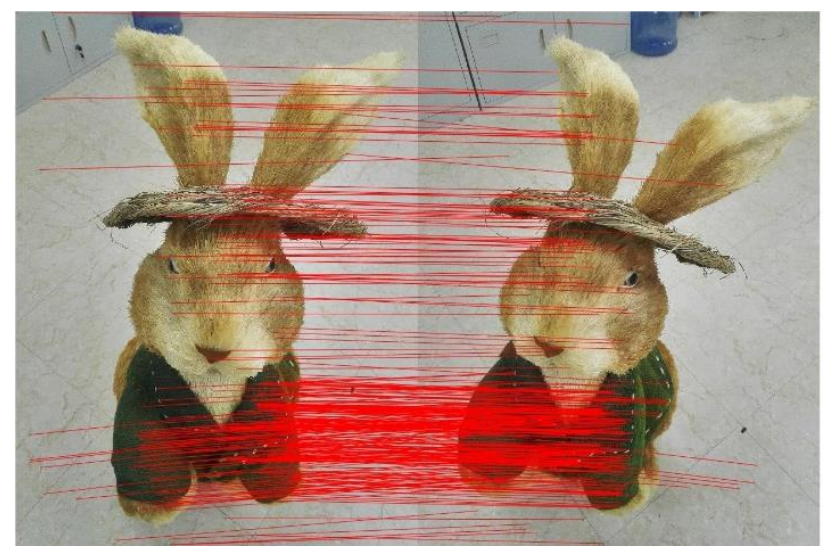

(a) Initial matching point connection diagram

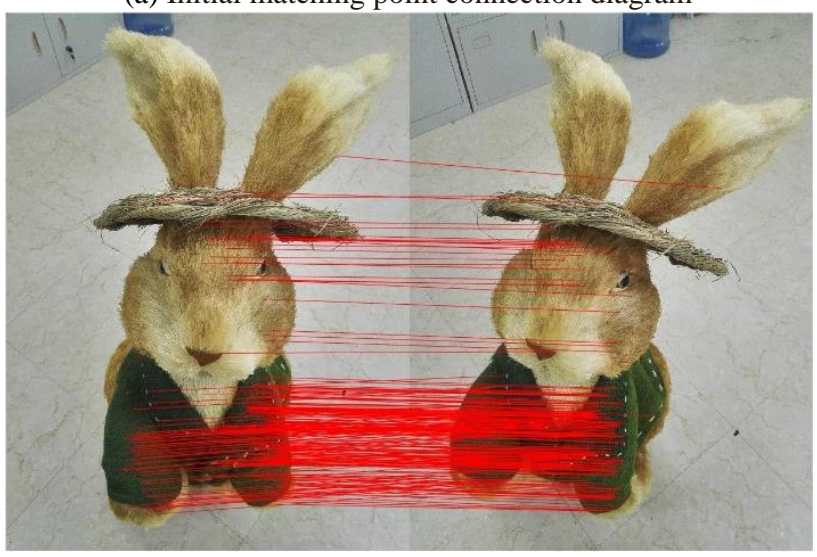

(b) Matching points after RANSAC algorithm processing

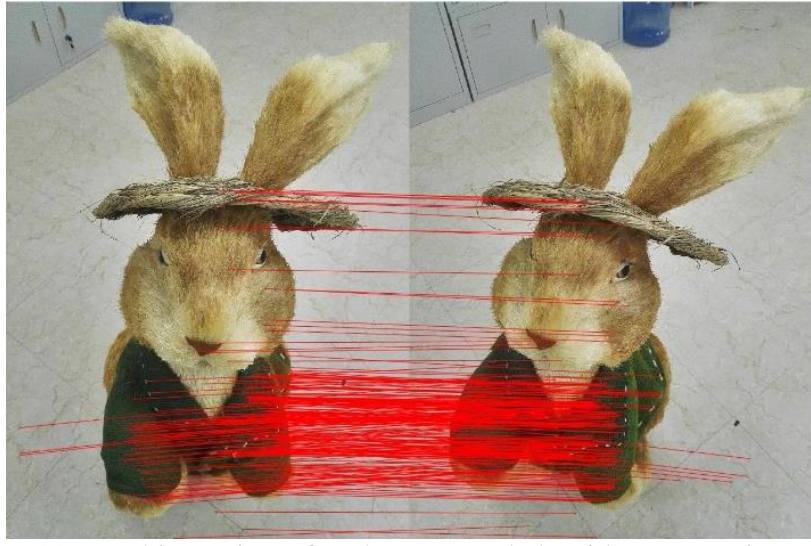

(c) Matching points after the proposed algorithm processing Figure 5. Corresponding point connection after preliminary feature matching (a) and screening algorithm processing, and the RANSAC algorithm (b) and the proposed algorithm (c) are used respectively.

\begin{tabular}{lccc}
\hline Algorithm & $\begin{array}{c}\text { Number } \\
\text { of Inliers }\end{array}$ & $\begin{array}{c}\text { Time } \\
\text { Consumption(s) }\end{array}$ & $\begin{array}{c}\text { Residual } \\
\text { MSE(pixels) }\end{array}$ \\
\hline $\begin{array}{l}\text { Proposed } \\
\text { Algorithm }\end{array}$ & 303 & 0.2042 & 1.1308 \\
RANSAC & 283 & 0.3845 & 1.1568 \\
\hline
\end{tabular}

Table 1 Comparison of the results of the two algorithms

The points screened by the two methods are connected separately, and it is found that the two methods successfully eliminate most of the mismatch points. Since the number of homonymy points cannot be accurately counted, the residual mean square error is used to characterize the algorithm error. And the RANSAC algorithm uses an average of 50 experiments. The results show that the errors calculated by the two methods are basically the same, but the proposed algorithm is more efficient. At the same time, considering the randomness of the RANSAC method, the computational stability of the proposed algorithm is better and the experimental results can be reproduced.

\section{DISCUSSION}

Traditional corresponding points screening method distinguishes the inliers and the outliers by one-dimensional residuals, which is not strict. The residual is not separable in one-dimensional space and there is often an overlap. As a result, some mismatches are divided into inlier sets, and some inliers are divided into outliers.

In order to solve this problem, the Gaussian kernel fuzzy clustering method is proposed in this paper, which maps the residual into the high-dimensional feature space by Gaussian kernel function. Since the residual is always finite, there should be a high-dimensional space that can linearly divide the residual into two categories. The cluster center position can be calculated by fuzzy clustering, and the separability judgment function is used to check whether the classification is optimal.

The advantage of the proposed method is the ability to discern the outliers with small residuals and inliers with large residuals. Experiments show that the method can effectively process the corresponding data whether it is adding wrong matching points or noise. Consider the requirements of points matching in the $3 \mathrm{D}$ 
reconstruction process. It is necessary to find the most suitable polar geometry relationship in calculating fundamental matrix. Even if the outliers with small residuals are retained, an accurate fundamental matrix can be obtained. However, for the reconstruction process, only the exact matching points can restore the correct three-dimensional coordinates, so that the proposed method is needed.

\section{CONCLUSION}

In this paper, the Gaussian kernel mapping method for polar geometric residuals is proposed. The one-dimensional space indistinguishable data is mapped to the high-dimensional feature space, thereby achieving linear separability. The fuzzy clustering is used to divide the residuals into two categories, and the separability function is used to judge whether it can be accurately divided into inliers and outliers.

Through the test experiments of simulated data and actual data, it can be concluded that compared with the traditional RANSAC algorithm, this proposed algorithm has obvious advantages in classification accuracy, efficiency and robustness. Although it does not effectively improve the computational accuracy of the fundamental matrix, it contributes to overall reconstruction accuracy.

\section{REFERENCES}

Brandt, S., 2002. Maximum likelihood robust regression with known and unknown residual models. Proc. SMVP, in conjunction with ECCV 2002, 97-102.

Brandt, S.S., 2006. Maximum likelihood robust regression by mixture models. Journal of Mathematical Imaging and Vision, 25(1), 25-48.

Duda, R.O., Hart, P.E., Stork, D.G., 2012. Pattern classification. John Wiley \& Sons.

Fischler, M.A., Bolles, R.C., 1981. Random sample consensus: a paradigm for model fitting with applications to image analysis and automated cartography. Communications of the ACM, 24(6), 381-395.

Goncalves, H., Corte-Real, L., Goncalves, J.A., 2011. Automatic image registration through image segmentation and SIFT. IEEE Transactions on Geoscience and Remote Sensing, 49(7), 2589-2600.

Hossein-Nejad, Z., Nasri, M., 2017. RKEM: Redundant Keypoint Elimination Method in Image Registration. IET Image Processing, 11(5), 273-284.

Jian, B., Vemuri, B.C., 2010. Robust point set registration using gaussianmixture models. IEEE transactions on pattern analysis and machine intelligence, 33(8),1633-1645.

Li , Y., Li, G., Gu, S., Long, K., 2016. Image stitching algorithm based on region block and scaleinvariant feature transform. Optics and Precision Engineering, 24(5),1197-1205.

Michaelsen, E., von Hansen, W., Kirchhof, M., et al., 2006. Estimating the essential matrix: Goodsac versus ransac. Photogrammetric Computer Vision, 1-6.

Xiong, H., Szedmak, S., Piater, J., 2013. A study of point cloud registration with probability product kernel functions. International Conference on 3D Vision-3DV. IEEE, 207-214.
Ye, Y., Shan, J., 2014. A local descriptor based registration method for multispectral remote sensing images with non-linear intensity differences. ISPRS Journal of Photogrammetry and Remote Sensing, 90, 83-95. 